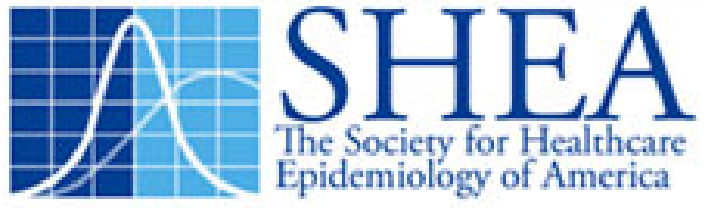

"The Dirty Hand in the Latex Glove": A Study of Hand Hygiene Compliance When Gloves Are Worn

Author(s): Christopher Fuller, MSc; Joanne Savage, MSc; Sarah Besser, MSc; Andrew Hayward, MD; Barry Cookson, FRCPath; Ben Cooper, PhD; Sheldon Stone, MD

Reviewed work(s):

Source: Infection Control and Hospital Epidemiology, Vol. 32, No. 12 (December 2011), pp. 1194-1199

Published by: The University of Chicago Press on behalf of The Society for Healthcare Epidemiology of America

Stable URL: http://www.jstor.org/stable/10.1086/662619

Accessed: 19/09/2012 08:47

Your use of the JSTOR archive indicates your acceptance of the Terms \& Conditions of Use, available at http://www.jstor.org/page/info/about/policies/terms.jsp

JSTOR is a not-for-profit service that helps scholars, researchers, and students discover, use, and build upon a wide range of content in a trusted digital archive. We use information technology and tools to increase productivity and facilitate new forms of scholarship. For more information about JSTOR, please contact support@jstor.org. 


\title{
"The Dirty Hand in the Latex Glove": A Study of Hand Hygiene Compliance When Gloves Are Worn
}

\author{
Christopher Fuller, MSc; Joanne Savage, MSc; ${ }^{1}$ Sarah Besser, MSc; ${ }^{2}$ Andrew Hayward, MD; ${ }^{1}$ \\ Barry Cookson, FRCPath; ${ }^{3}$ Ben Cooper, $\mathrm{PhD} ;{ }^{4}$ Sheldon Stone, $\mathrm{MD}^{5}$
}

\begin{abstract}
B ACKGROUND AND OвJECTIVE. Wearing of gloves reduces transmission of organisms by healthcare workers' hands but is not a substitute for hand hygiene. Results of previous studies have varied as to whether hand hygiene is worse when gloves are worn. Most studies have been small and used nonstandardized assessments of glove use and hand hygiene. We sought to observe whether gloves were worn when appropriate and whether hand hygiene compliance differed when gloves were worn.
\end{abstract}

DESIGN. Observational study.

PARTicipants AND SETting. Healthcare workers in 56 medical or care of the elderly wards and intensive care units in 15 hospitals across England and Wales.

Methods. We observed hand hygiene and glove usage (7,578 moments for hand hygiene) during 249 one-hour sessions. Observers also recorded whether gloves were or were not worn for individual contacts.

RESUlTs. Gloves were used in 1,983 (26.2\%) of the 7,578 moments for hand hygiene and in 551 (16.7\%) of 3,292 low-risk contacts; gloves were not used in $141(21.1 \%)$ of 669 high-risk contacts. The rate of hand hygiene compliance with glove use was $41.4 \%$ ( 415 of 1,002 moments), and the rate without glove use was 50.0\% (1,344 of 2,686 moments). After adjusting for ward, healthcare worker type, contact risk level, and whether the hand hygiene opportunity occurred before or after a patient contact, glove use was strongly associated with lower levels of hand hygiene (adjusted odds ratio, 0.65 [95\% confidence interval, $0.54-0.79$ ]; $P<.0001$ ).

CONCLUSion. The rate of glove usage is lower than previously reported. Gloves are often worn when not indicated and vice versa. The rate of compliance with hand hygiene was significantly lower when gloves were worn. Hand hygiene campaigns should consider placing greater emphasis on the World Health Organization indications for gloving and associated hand hygiene.

trial registration. National Research Register N0256159318.

Infect Control Hosp Epidemiol 2011;32(12):1194-1199

Wearing of gloves reduces the likelihood of contamination of healthcare workers' hands after patient contact ${ }^{1-3}$ and thus the potential transmission of pathogens between patients. ${ }^{2,4}$ World Health Organization (WHO) guidelines recommend use of gloves when contact with body fluids is anticipated and when patients are to be managed with contact precautions. ${ }^{4}$ However, gloves are not a substitute for effective hand hygiene: they do not eliminate contamination of hands, and they are themselves potential vectors of transmission between patients if not changed between contacts. ${ }^{1,3,5}$ WHO guidelines, ${ }^{4}$ therefore, state that gloves should be changed between patient contacts and that hands should be cleaned before putting on gloves (when a moment for hand hygiene occurs) and immediately after removing gloves.
Healthcare workers' compliance with WHO guidelines may be poor, with gloves being worn when not required and vice versa. ${ }^{5-7}$ Some investigators report lower rates of hand hygiene compliance if gloves are worn, ${ }^{5,6,8}$ while others report unchanged or better rates of compliance. ${ }^{9-14}$ It is hard to know how representative such studies are, as they vary in size, methodology, indications for glove use, and the numbers of wards or hospitals studied, with most taking place in only one hospital. We therefore carried out a study of glove use and associated hand hygiene behaviors on 56 wards in 15 hospitals in England and Wales to observe whether gloves were worn when indicated and to determine whether the rate of hand hygiene compliance was different when gloves were or were not worn.

Affiliations: 1. University College London (UCL) Research Department of Infection and Population Health, UCL (Hampstead Campus), Royal Free Hospital, London, United Kingdom; 2. King's Centre for Risk Management, King's College London, London, United Kingdom; 3. Laboratory of Healthcare Associated Infection, Centre for Infections, Health Protection Agency, Colindale, London, United Kingdom; 4. Mahidol-Oxford Tropical Medicine Research Unit (MORU), Bangkok, Thailand; 5. Health Services for Elderly People, Royal Free Hospital NHS Trust, London, United Kingdom.

Received February 10, 2011; accepted July 26, 2011; electronically published October 17, 2011.

(C) 2011 by The Society for Healthcare Epidemiology of America. All rights reserved. 0899-823X/2011/3212-0007\$15.00. DOI: 10.1086/662619 
TABLE 1. Proportion of Moments for Hand Hygiene at Which Healthcare Workers (HCWs) Wore Gloves

\begin{tabular}{lc}
\hline Type of contact & Proportion (\%) of moments \\
\hline All & $1,983 / 7,578(26.2)$ \\
By location & $675 / 2,305(29.3)$ \\
Intensive therapy unit & $1,308 / 5,273(24.8)$ \\
ACE/GM ward & \\
By risk category & $528 / 669(78.9)$ \\
High-risk contacts & $551 / 3,292(16.7)$ \\
Low-risk contacts & $904 / 3,617(25.0)$ \\
Unobserved contacts & \\
By class of practitioner & $1,593 / 5,111(31.2)$ \\
Nurse & $54 / 1,202(4.5)$ \\
Doctor & $336 / 1,265(26.6)$ \\
Other HCW
\end{tabular}

note. ACE/GM; acute care of the elderly and general medical.

\section{METHODS}

A series of 1-hour-long covert observations of hand hygiene and glove usage were undertaken at 6-week intervals in each of a convenience sample of 56 wards in 15 hospitals across England and Wales. Wards were taking part in a national, stepped-wedge, cluster-randomized, controlled trial of a feedback intervention, the Feedback Intervention Trial (FIT), which had commenced in October 2006.

In each hospital, 1 intensive therapy unit and a maximum of 3 acute care of the elderly and general medical (ACE/GM) wards had previously been recruited into the FIT study. Sites were recruited by requests posted on the Web site of the English and Welsh "cleanyourhands" campaign ${ }^{15}$ and by contacting infection control teams directly. Hospitals recruited included 2 teaching and 13 district general hospitals with 400-1,200 beds.

The aim of the FIT intervention was to improve the hand hygiene compliance of healthcare workers in ACE/GM wards and in intensive therapy units. Improving compliance with appropriate glove usage was not part of the study intervention. On the basis of the goal setting ${ }^{16}$ and control ${ }^{17}$ theories, the FIT intervention was delivered weekly to individual ward staff and groups as part of an audit cycle repeated every 4 weeks. Observation of hand hygiene behavior and feedback were coupled to personalized goal setting and action planning. Although all 56 wards had been allocated to use the intervention and had received training in how to implement the FIT, only 33 (59\%) had ever carried out any part of the intervention.

Covert observations of hand hygiene had been carried out since October 2006 on all participating wards by research assistants who had been trained to use the observation tool, as described elsewhere. ${ }^{18}$ Between January 2009 and November 2009, glove use was also recorded over a total of 249 hours on 15 intensive therapy units and 41 ACE/GM wards (for a total of 7,578 moments for hand hygiene).

Observations were undertaken in open ward areas ("bays") that were shared by 4-6 patients but not for patients under contact precautions in single-room accommodation. This was for reasons of patient privacy and to reduce Hawthorne effects. Patients under contact precautions in an open bay area were observed, but this was a rare occurrence and not specifically documented.

Hand hygiene was recorded using a rigorously standardized and validated hand hygiene observation tool: HHOT version $1 .^{19,20}$ Data collection was modified slightly, for the purposes of this study, to record glove use. Although the version of the HHOT used for the study was developed before the publication of the WHO " 5 moments for hand hygiene," standard operating procedures are fully consistent with the WHO guidelines. In this study we use the WHO term hand hygiene "moment," instead of the original term hand hygiene "opportunity" used in the first version of the HHOT, to help readers familiar with WHO terminology.

The HHOT version 1 records the following information:

1. The type of healthcare worker observed.

2. Whether the indication for hand hygiene (corresponding to the WHO hand hygiene "moment") took place before or after patient contact.

3. The risk level: "high risk," corresponding to the WHO categories "aseptic task" or "body fluid contact"; "low risk," corresponding to the WHO categories "direct patient contact" or "contact with the patients' environment"; and "unobserved contact," which does not correspond to any WHO category but records instances in which a healthcare worker attends a patient behind the bedside curtains. In these latter circumstances a contact cannot be seen but is inferred and the corresponding hand hygiene behavior can sometimes be observed. The unobserved contact category allows the reporting of the maximum number of observed hand hygiene behaviors and generates an overall compliance rate, the main purpose of the HHOT. For the purposes of this study, inclusion of unobserved contacts facilitated maximum reporting of glove usage.

4. The type of hand hygiene behavior or cleaning agent used: "alcohol hand rub," "soap," "no action," or "unknown." The latter term was used for moments for which it was not possible to identify whether hands were cleaned or not.

For the purposes of this study, the original HHOT version $1^{20}$ was modified to record only whether gloves were worn for individual hand hygiene moments and associated hand hygiene. If the moment was unobserved but the healthcare worker was seen to be wearing gloves, glove use was recorded. An example of this was when a healthcare worker was seen to don gloves before going behind a patient's bedside curtains. The tool was not able to differentiate between instances in which gloves were removed but hands were not cleaned and instances in which the same pair of gloves was used for multiple contacts.

The proportion of each type of contact in which gloves were worn was recorded, as was hand hygiene compliance for each type of contact with and without gloves. The null 
TABLE 2. Rates of Compliance with Hand Hygiene When Gloves Were Worn and When Gloves Were Not Worn

\begin{tabular}{lrrr}
\hline & \multicolumn{2}{c}{$\begin{array}{c}\text { Proportion (\%) of moments } \\
\text { with hand hygiene compliance }\end{array}$} & \\
\cline { 2 - 3 } & $\begin{array}{c}\text { When gloves } \\
\text { were worn }\end{array}$ & $\begin{array}{c}\text { When gloves } \\
\text { were not worn }\end{array}$ & \multicolumn{1}{c}{ RR (95\% CI) } \\
\hline Type of moment & $415 / 1,002(41.4)$ & $1,344 / 2,686(50.0)$ & $0.83(0.76-0.90)$ \\
\hline $\begin{array}{l}\text { All } \\
\text { By location }\end{array}$ & & & \\
$\quad$ Intensive therapy unit & $246 / 514(47.9)$ & $488 / 896(54.5)$ & $0.88(0.79-0.98)$ \\
$\quad$ ACE/GM ward & $169 / 488(34.6)$ & $856 / 1,790(47.8)$ & $0.72(0.64-0.83)$ \\
By risk level & & & \\
$\quad$ High-risk contact & $213 / 484(44.0)$ & $72 / 123(58.5)$ & $0.75(0.63-0.90)$ \\
$\quad$ Low-risk contact & $203 / 518(39.2)$ & $1,272 / 2,563(49.6)$ & $0.79(0.70-0.89)$ \\
By timing & & & \\
$\quad$ Before contact & & & \\
$\quad$ After contact & $98 / 330(29.7)$ & $170 / 424(40.1)$ & $0.74(0.60-0.91)$ \\
\hline
\end{tabular}

Nоте. ACE/GM; acute care of the elderly and general medical; $\mathrm{CI}$, confidence interval; $\mathrm{RR}$, risk ratio.

hypothesis was that there would be no difference in hand hygiene compliance between moments when gloves were worn and moments when gloves were not worn.

The proportion of hand hygiene moments for which gloves were used was also expressed as a percentage. Of the 7,578 hand hygiene moments, 3,890 were excluded from the main analysis $(3,617$ contacts classed as unobserved and 273 hand hygiene behaviors classed as unknown). In these instances, it was not possible to identify the nature of the contact and/ or whether hands had been cleaned. Confidence intervals for risk ratios and odds ratios were calculated using normal approximation. Adjusted odds ratios were calculated using a generalized linear mixed model for binomial outcomes (hand hygiene was recorded as either yes or no), fitted using the Laplace approximation and entering all variables. Random intercepts were used to account for clustering at the hospital and ward level (wards nested within hospitals). Statistical analysis was performed with $\mathrm{R}$ software, version $2.9{ }^{22}$

\section{RES ULT S}

Table 1 shows the proportions of hand hygiene moments for which gloves were used or were not used. Overall, gloves were used in 1,983 (26.2\%) of 7,578 moments; the proportion was slightly higher in intensive therapy units than in ACE/GM wards. The rate of glove use was lower among doctors, at just $4.5 \%$ of moments $(54$ of 1,202$)$, than among nurses, at $31.2 \%$ of moments $(1,593$ of 5,111$)$. Gloves were used in 551 $(16.7 \%)$ of 3,292 low-risk contacts, when there would not have been a clinical indication for using gloves. Conversely, gloves were not used in 141 (21.1\%) of 669 high-risk contacts, when their use would have been indicated. Gloves were used in $904(25.0 \%)$ of 3,617 unobserved contacts (ie, healthcare workers were observed to be wearing gloves on entering or exiting patients curtains).

Overall hand hygiene compliance whether gloves were worn or not was $53.6 \%(2,795$ of 5,214). When unobserved contacts were removed from the analysis hand hygiene compliance was slightly lower, at $47.7 \%(1,759$ of 3,688$)$. Table 2 compares hand hygiene compliance for moments when gloves were worn and moments when they were not worn for the 3,688 hand hygiene moments for which the hand hygiene behavior was known and the contact was observed. The rate of compliance was significantly lower when gloves were worn, both overall and in all subgroups defined by ward type, timing of hand hygiene moment (before or after patient contact), and contact risk level. Adjusting for all these factors, healthcare worker type and ward and hospital clustering effects confirmed a strong association between glove use and reduced hand hygiene compliance (Table 3 ).

\section{I S C USS I O N}

The 2 main findings of this study are, first, that glove use often did not comply with WHO standards-gloves were worn when not indicated and vice versa-and, second, that the rate of compliance with hand hygiene was significantly worse when gloves were worn. This finding has possible important clinical implications for the control of organisms such as methicillin-resistant Staphylococcus aureus (MRSA), given that wearing gloves is a major component of contact precautions, which in many instances may be the only isolation method for patients because of a shortage of isolation facilities. $^{23}$

The strengths of this study are its large size and its use of a rigorously standardized hand hygiene observation tool (HHOT) with clearly defined standard operating procedures. It is the largest study of glove use and hand hygiene compliance of which we are aware, not only recording a large number of hand hygiene moments but also recording practice over a large national sample of wards and hospitals across England and Wales. The study's pragmatic sample and design 
TA B LE 3. Adjusted Odds Ratios (ORs) for Factors Associated with Hand Hygiene Compliance Estimated from the Generalized Linear Mixed Model

\begin{tabular}{lcc}
\hline Factor & Adjusted OR (95\% CI) & $P$ \\
\hline Gloves worn & $0.65(0.54-0.79)$ & $<.0001$ \\
$\begin{array}{l}\text { Intensive therapy unit } \\
\quad \text { location }\end{array}$ & $1.25(0.96-1.63)$ & .10 \\
High-risk contact & $1.34(1.07-1.68)$ & .01 \\
After contact & $2.02(1.69-2.41)$ & $<.0001$ \\
Nurse $^{\mathrm{a}}$ & $2.21(1.66-2.94)$ & $<.0001$ \\
Other HCW $^{\mathrm{b}}$ & $1.05(0.76-1.44)$ & .78 \\
\hline
\end{tabular}

NOTE. CI, confidence interval; HCW, healthcare worker.

a Compared with doctors and other HCWs.

${ }^{\mathrm{b}}$ Compared with nurses and doctors.

gives a geographically and clinically representative picture of practice in England and Wales.

Among the limitations of the study are that the HHOT provides an incomplete assessment of gloving practice, not having been designed for this purpose. It was easy to amend the tool to record whether gloves were worn or not for a patient contact or hand hygiene moment, although we did not formally assess the interrater reliability of this simple amendment. We found it impractical, however, to record whether gloves were changed between patient contacts while simultaneously documenting hand hygiene behavior. Assessment of gloving practice was further limited because observers were unable, for the reasons described above, to assess it for healthcare workers caring for patients isolated in side rooms under contact precautions, which is itself an indication for glove use, and were unable to go behind the curtains to observe healthcare worker care of patients. There is no comprehensive, validated, reliable measure of glove use reported in the literature. Two implications of our study are that such a measure ought to be developed and that assessment of gloving and associated hand hygiene compliance may be best carried out separately from standard audits of hand hygiene compliance.

Another limitation that possibly affects the generalizability of our findings is that the wards included in the study were drawn from those enrolled in a randomized controlled trial. The practice of both hand hygiene and glove usage on these wards might be better either by virtue of having enough interest in hand hygiene to volunteer for an intervention trial or by virtue of the intervention itself. Although the intervention covered appropriate hand hygiene compliance in association with glove usage, it did not focus on this in particular, nor did it cover appropriate use of gloves. In addition, although all wards had been allocated to use the intervention, a significant proportion (41\% [23 of 56]) had not yet started the intervention. Although it is possible to suggest that the overall rate of hand hygiene compliance was better than the national average in study wards, there is no reason to think that the intervention would change the overall relationship between glove use and hand hygiene, and we felt that it was unlikely to bias the study results toward reduced compliance with hand hygiene in association with glove wearing.

It is hard to compare the findings of this study with those of other studies in the literature. It is a much larger study (more wards and hospitals) than any other of which we are aware, and different investigators have used different definitions in the indications for glove use and the assessment of hand hygiene in association with glove wearing. Most studies have only assessed hand hygiene compliance after patient contact, ${ }^{5,6,9,10,12,24}$ while others have used indications for glove use that differed from the WHO guidelines. ${ }^{12,14}$ Our study excluded patients under contact precautions housed in side rooms, although not those housed in ward bays, whereas other studies included many such patients. ${ }^{5,9}$

The rate of glove use in our study was unusually low, at $26 \%$ of moments for hand hygiene $(1,983$ of 7,578$)$, compared with the $40 \%-93 \%$ of moments reported in most studies. ${ }^{6,7,9,11,14}$ A possible explanation may be that the culture of hand hygiene in England and Wales has changed, relying less on gloves and more on use of alcohol-based hand rub and soap, whose use has been prioritized by the national "cleanyourhands" campaign in 2004-2009..$^{15,25,26}$ Initial campaign documents made no mention of glove use and the associated hand hygiene behaviors expected, ${ }^{27}$ although subsequent campaign materials drew attention to the WHO glove use guideline. ${ }^{28}$ This may explain why we report the rate of glove use to be low, except during high-risk contacts. The rate of glove use by doctors observed in this study is especially low: gloves were worn for just $4.5 \%$ of hand hygiene moments (54 of 1,202). The reasons for this are unclear, but it may be a reflection of the types of patient contacts carried out by doctors.

The overall rate of hand hygiene compliance was disappointingly low, at just $47.7 \%$ of hand hygiene moments. Even so, this compares favorably with much of the published literature, which suggests a figure of around $40 \%{ }^{29}$ The large sample size and geographical and clinical range of the study wards is likely to mean that this estimate is representative of the true picture in the UK National Health System.

Despite the differences in study size, sample size, and methods, other studies have also found that the rate of compliance with hand hygiene is worse when gloves are worn. ${ }^{5,6,8}$ For instance, a study carried out in wards in a general hospital in Spain $^{8}$ reported that wearing gloves was an important risk factor associated with failure to perform hand hygiene (odds ratio, 2.2), a finding that is similar to ours. However, ours is the first study, to our knowledge, to have reported this as a consistent finding for all types of hand hygiene moments in a large national sample of hospitals and wards.

It is unclear why glove use should be associated with poorer compliance with hand hygiene, and it has been suggested that glove use and performance of hand hygiene are 2 distinct behaviors with distinct behavioral determinants. ${ }^{7}$ Healthcare workers may feel that wearing gloves not only protects them 
from the pathogens on patients but also protects patients from the pathogens on healthcare workers' hands ${ }^{30,31}$ and that this obviates the need for hand disinfection. Hand hygiene behavior has been classified as being either inherent (motivated by feelings of disgust, after contact with dirt or perceived dirt) or elective (not motivated by disgust). ${ }^{32}$ No such analysis of gloving behavior has been done, but it may be that gloving follows the inherent pattern but is perceived to provide enough protection to be a substitute for hand disinfection. This study suggests that far from conferring protection, the reality is that healthcare workers do not always clean their hands before donning gloves, that their hands pick up further organisms during high-risk contacts, and that hands are not always cleaned when the gloves are removed. This results in what may be termed "the dirty hand in the latex glove." Further study of the behavioral determinants of glove use and its relationship to hand hygiene are warranted, especially in light of these findings.

It is of particular concern that donning gloves for highrisk contacts is associated with poor hand hygiene. Since the rate of glove usage recorded in our study is much lower than that reported by other investigators, we would not go as far as some in suggesting that glove use is the foundation of poor hand hygiene compliance. ${ }^{5}$ We would, however, suggest that improving the rate of hand hygiene compliance in association with wearing gloves could be critical in raising compliance levels above the threshold required to reduce the transmission of infection. Modeling studies of, for example, transmission of MRSA suggest that very small increments of increase in the rate of hand hygiene compliance, especially when the baseline is around $40 \%$, could have major effects on reducing transmission, with relatively little extra effect once the rate of compliance exceeds $50 \%$. $^{32,33}$

Although this study did not include observations of patients under contact precautions, our findings could also have implications for the care of patients colonized or infected with antimicrobial-resistant organisms. A recent study modeling the effectiveness of contact precautions found that although glove and apron use was likely to reduce the rate of transmission of MRSA by around $28 \%$, there was a considerable degree of uncertainty around this estimate; in fact, there was a $30 \%$ chance that contact precautions may increase the rate of transmission..$^{35}$ The reduced rate of compliance with hand hygiene associated with glove use provides a potential explanation for this. This leads to the potential paradox that, for those contacts when gloves are most likely to be worn (ie, contacts with patients known to carry a resistant organism) and hand hygiene is of particular importance, hand hygiene is less likely to take place.

Indeed, if the hand hygiene movement were to focus now on limiting glove use to high-risk contacts (ie, aseptic tasks, body fluid contact, or contacts with isolated patients) and insisting that hand hygiene be performed before and after such moments, the overall rate of compliance would, on the basis of our figures, rise from $47.7 \%$ to $57.9 \%$ of moments.
This could be highly significant in reducing the reproduction number to levels associated with very low endemic levels of MRSA colonization and infection, even if the transmissibility of an organism was as high as $20 \%$ (the highest level assumed in some models). ${ }^{34}$ This might prove a more effective hand hygiene improvement strategy than aiming for $90 \%-100 \%$ overall compliance. National and international campaigns, such as the English and Welsh "cleanyourhands" campaign and the WHO "Clean Care Is Safer Care" campaign, should consider emphasizing better gloving practice and associated hand hygiene.

In conclusion, data we obtained for a large national sample of wards and hospitals shows that although the rate of glove use is relatively low, gloves are still often worn when not indicated and vice versa. The rate of compliance with hand hygiene is significantly worse if gloves are worn for all types of hand hygiene moments, especially high-risk patient contacts and before patient contacts. Raising the rate of compliance with hand hygiene when gloves are worn to the levels observed when gloves are not worn might only have a small effect on the overall rate of hand hygiene compliance but could have a major effect on the transmission of infection. This is all the more likely if future studies were to show that this finding holds for contacts with patients known to be colonized or infected with an antimicrobial-resistant organism, when glove use is recommended. A validated comprehensive assessment of glove use and its associated hand hygiene behaviors is needed. Study of the behavioral and psychological predictors of gloving behavior and its relationship to hand disinfection is warranted and would be useful to help design an intervention to improve the rate of hand hygiene compliance when gloves are worn and to limit glove use to those indications specified in the WHO guidelines.

\section{ACKNOWLEDGMENTS}

Financial support. This study was funded by the Patient Safety Research Programme, GOJO Industries, and the Royal Free Hospital Trustees.

Potential conflicts of interest. All authors report no conflicts of interest relevant to this article.

Address reprint requests to Christopher Fuller, Nursing Research Fellow, University College London, Research Department of Infection and Population Health, UCL (Hampstead Campus), Royal Free Hospital, London NW3 2PF, United Kingdom (christopher.fuller@ucl.ac.uk).

\section{REFERENCES}

$\rightarrow$ 1. Pittet D, Dharan S, Touveau S, Savan V, Perneger TV. Bacterial contamination of the hands of hospital staff during routine patient care. Arch Intern Med 1999;159:821-826.

$\rightarrow$ 2. Johnson S, Gerding DN, Olson MM, et al. Prospective, controlled study of vinyl glove use to interrupt Clostridium difficile nosocomial transmission. Am J Med 1990;88(2):137-140.

$\rightarrow$ 3. Snyder GM, Thom KA, Furono JP, et al. Detection of methicillinresistant Staphylococcus aureus and vancomycin-resistant enter- 
ococci on the gowns and gloves of healthcare workers. Infect Control Hosp Epidemiol 2008;29:583-589.

4. World Health Organization (WHO). World Health Organization Guidelines on Hand Hygiene. Geneva: WHO, 2009.

$\rightarrow$ 5. Girou E, Chaia SHT, Oppeina F, et al. Misuse of gloves: the foundation for poor compliance with hand hygiene and potential for microbial transmission? J Hosp Infect 2004;57:162-169.

6. Flores A, Pevalin D. Healthcare workers compliance with glove use and the effect of glove use on compliance with hand hygiene. J Infect Prevent 2006;7:15-18.

$\rightarrow$ 7. Hitoto H, Kouatchet A, Dubé L, et al. Factors affecting compliance with glove removal after contact with a patient or environment in four intensive care units. J Hosp Infect 2009;71: 186-188.

$\rightarrow$ 8. Sánchez-Payá J, Galicia-García MD, Gracia-Rodríguez RM, et al. Compliance with hand hygiene guidelines and determinants of compliance [abstract]. Enferm Infecc Microbiol Clin 2007;25: 365-368.

$\rightarrow$ 9. Kim PW, Roghmann M, Perencevich EN, Harris AD. Rates of hand disinfection associated with glove use, patient isolation, and changes between exposure to various body sites. Am J Infect Control 2003;31:97-103.

$\rightarrow$ 10. Lund S, Jackson J, Leggett J, Hales L, Dworkin R, Gilbert D. Reality of glove use and handwashing in a community hospital. Am J Infect Control 1994;22:352-357.

$\rightarrow$ 11. Lankford MG, Zembower TR, Trick WE, Hacek DM, Noskin GA, Peterson LR. Influence of role models and hospital design on hand hygiene of healthcare workers. Emerg Inf Dis 2003;9: 217-223.

$\rightarrow$ 12. Zimakof J, Stormark M, Olesen Larsen S. Use of gloves and handwashing behaviour among healthcare workers in intensive care units: a multicentre investigation in four hospitals in Denmark and Norway. J Hosp Infect 1993;24:63-67.

$\rightarrow$ 13. Trick WE, Vernon MO, Welbel SF, DeMarais P, Hayden MK, Weinstein RA. 2007. Multicenter intervention program to increase adherence to hand hygiene recommendations and glove use to reduce the incidence of antimicrobial resistance. Infect Control Hosp Epidemiol 2007;28:42-49.

$\rightarrow$ 14. Pan A, Mondello P, Posfay-Barbe K, et al. Hand hygiene and glove use behavior in an Italian hospital. Infect Control Hosp Epidemiol 2007;28:1099-1102.

15. National Health Service National Patient Safety Agency. cleanyourhands Web site. http://www.npsa.nhs.uk/cleanyourhands/. Accessed June 7, 2010.

16. Locke E, Latham G. A Theory of Goal Setting and Task Performance. Englewood Cliffs, NJ: Prentice Hall, 1990.

17. Carver CS, Scheier MF. On the structure of behavioral selfregulation. In: Boekaerts M, Pintrich PR, Zeidner M, eds. Handbook of Self-Regulation. San Diego: Academic Press, 2000.

$\rightarrow$ 18. Fuller C, Besser S, Cookson BD, et al. Technical note: assessment $\rightarrow$ of blinding of hand hygiene observers in randomized controlled trials of hand hygiene interventions. Am J Infect Control 2010; 38:332-334.

$\rightarrow$ 19. McAteer J, Stone S, Fuller C, et al; the NOSEC/FIT group. Development of an observational measure of healthcare worker hand hygiene behaviour: the hand hygiene observation tool (HHOT). J Hosp Infect 2008;68:222-229.

20. NOSEC: national observation study of the effectiveness of the cleanyourhands campaign, January 2005-December 2009. http: //www.idrn.org/nosec.php. Accessed May 10, 2011.

21. My 5 moments for hand hygiene. http://www.who.int/gpsc/ 5may/background/5moments/en/index.html. Accessed June 7, 2010.

22. Team RDC. R: A Language and Environment for Statistical Computing. Vienna: R Foundation for Statistical Computing, 2007.

23. National Audit Office. Reducing Healthcare Associated Infections in Hospitals in England: Report by the Comptroller and Auditor General HC 560 Session 2008-9, 12 June 2009. London: HMSO, 2009. http://www.nao.org.uk/publications/ 0809/reducing_healthcare_associated.aSpx. Accessed September 21, 2009.

24. Whitby M, McLaws ML. Hand-washing in healthcare workers: accessibility of sink location does not improve compliance. $J$ Hosp Infect 2004;58:247-253.

25. Stone S, Slade R, Fuller C, et al. Early communication: does a national campaign to improve hand hygiene in the NHS work? initial English and Welsh experience from the NOSEC study. $J$ Hosp Infect 2007;66:293-296.

26. Stone S, Fuller C, Slade R, et al. The success and effectiveness of the world's first national cleanyourhands campaign in England and Wales 2004-2008: final results. In: Program and abstracts of the 19th European Congress of Clinical Microbiology and Infectious Diseases; May 16-19, 2009; Helsinki.

27. National Patient Safety Agency (NPSA). Ready Steady Go: The Full Guide to Implementing the cleanyourhands Campaign in Your Trust. London: NPSA, 2004.

28. National Patient Safety Agency (NPSA). Flowing with the Go: The Complete Year Two Maintenance Handbook for cleanyourhands Partner Trusts. London: NPSA, 2009.

29. Erasmus V, Daha TJ, Brug H, et al. Systematic review of studies on compliance with hand hygiene guidelines in hospital care. Infect Control Hosp Epidemiol 2010;31:283-294.

30. Jang J-H, Wu S, Kirzner D, Moore C, et al. Focus group study of hand hygiene practice among healthcare workers in a teaching hospital in Toronto, Canada. Infect Control Hosp Epidemiol 2010; 31:144-150.

31. Wilcox G, Diekema D, Sherry DS, Herwaldt L. Healthcare workers' knowledge, beliefs and attitudes about hand hygiene performance. In: Program and abstracts of Fifth Decennial International Conference on Healthcare-Acquired Infections 2010; May 18-22, 2010; Atlanta.

32. Whitby M, Pessoa-Silva CL, McLaws ML, et al. Behavioural considerations for hand hygiene practices: the basic building blocks. J Hosp Infect 2007;65:1-8.

33. Cooper BS, Medley GF, Scott GM. Preliminary analysis of the transmission dynamics of nosocomial infections: stochastic and management effects. J Hosp Infect 1999;43:131-147.

34. Beggs CB, Shepherd SJ, Kerr KG. Increasing the frequency of hand washing by healthcare workers does not lead to commensurate reductions in staphylococcal infection in a hospital ward. BMC Infect Dis 2008;8:114.

35. Kypraios T, O’Neill PD, Huang SS, Rifas-Shiman SL, Cooper BS. Assessing the role of undetected colonization and isolation precautions in reducing methicillin-resistant Staphylococcus aureus transmission in intensive care units. BMC Infect Dis 2010; 10:29. 